LMU-12/97

UWTHPH-1997-36

TUM-HEP-294/97

October 97

\title{
Higher Dimensional Operators in Top Condensation from a Renormalization Group Point of View
}

\author{
Andreas Blumhofer円, Richard Dawid] And Johannes Manusq \\ a Sektion Physik, Ludwig-Maximilians-Universität München, \\ Theresienstr.37, D-80333 München, Germany \\ ${ }^{\mathrm{b}}$ Institut für Theoretische Physik, Universität Wien \\ Boltzmanngasse 5, A-1090 Wien, Austria \\ ${ }^{\mathrm{c}}$ Institut für Theoretische Physik, Technische Universität München, \\ James-Franck-Straße, D-85748 Garching, Germany
}

\begin{abstract}
The predictive power of top-condensation models strongly depends on the behaviour of higher dimensional operators. These are analyzed in this paper by an extension of the standard renormalization group $(\mathrm{RG})$ arguments which turns out to be a surprisingly powerful tool. Top-condensation models intermediated by underlying scalar exchange can be shown to be mere reparametrizations of the standard model. Further on, RG-arguments show that dynamical vector states cannot be lowered in top-condensation models. Finally we give a general argument concerning the size of higher dimensional operators of heavy vector exchange.
\end{abstract}

\footnotetext{
${ }^{a}$ Email: Blumhofer@Photon.HEP.Physik.Uni-Muenchen.DE

bEmail: Richard.Dawid@Merlin.PAP.Univie.AC.AT

${ }^{\mathrm{c}}$ Email: Johannes.Manus@Physik.TU-Muenchen.DE
} 


\section{Introduction}

Dynamical symmetry breaking is an alternative of spontaneous electro-weak symmetry breaking which replaces the fundamental Higgs field of the standard model by a composite scalar field. Instead of fundamental scalars the simplest model of top-condensation [3], 4] introduces a new four-Fermion interaction capable of forming the electro-weak symmetry breaking top-condensate円. The dynamics then generates an effective scalar sector which describes the symmetry breaking in analogy to the Ginzburg-Landau description of superconductivity.

It soon turned out that top-condensation requires a more specific picture of the underlying interaction. The works of Hasenfratz et al. [7] and Zinn-Justin [8] showed that every standard model scenario can be reparametrized as a top-condensation model if one has total freedom in the choice of higher dimensional operators. To control these higher dimensional operators several specific models of underlying interactions were constructed [9, 10, 13]. In this work we analyze the structure of higher dimensional operators in the framework of the renormalization group (RG) approach. We show, that a number of questions, which remained unsolved or only partially solved during the last years in the framework of a dynamical description, can be answered in an elegant way by a consequent use of RG-arguments.

Section 2 gives a short introduction to the $\mathrm{RG}$-formulation of top-condensation including higher dimensional operators. This method was introduced in one of the founding papers of that model by Bardeen, Hill and Lindner 4 to derive quantitative predictions of standard top-condensation. Its consistency with the improved dynamical description of the PagelsStokar formula was shown in [5]. Though the inclusion of higher dimensional operators was sketched e.g. in [13], the theoretical implications of this generalized approach were never really exploited. To do so will be the goal of this work.

Section 3 rephrases in a RG-framework the discussion about higher dimensional operators led in [7] and [8] which allows a very intuitive understanding of that problematics. After a general discussion of relevant and irrelevant operators from a RG-point of view in section 4, we have the tools to reach out to several new statements about the structure of top-condensation models. In section 5 it turns out that top condensation via heavy scalar exchange is an example of a pure standard model reparametrization. Section 6 shows that it is not possible to lower the scale of composite vector states in top-condensation models. Section 7 gives a $\mathrm{RG}$-argument against the existence of relevant higher dimensional operators stemming from

\footnotetext{
${ }^{1}$ For an overview see e.g. ref. [6]
} 
heavy vector exchange. After a comparison of our RG-arguments with dynamical methods in section 8 we draw our conclusions.

\section{Renormalization Group Approach}

The simplest realization of the idea of top-condensation [2, 3, 4], is a Nambu-Jona-Lasinio [1] like model, consisting of the kinetic parts of the ordinary quarks, leptons and $S U(3)_{c} \times$ $S U(2)_{L} \times U(1)_{Y}$ gauge fields and a new attractive four-Fermion interaction. The Lagrangian isf

$$
\mathcal{L}=\mathcal{L}_{\text {kin }}+G \bar{\psi}_{L} t_{R} \bar{t}_{R} \psi_{L}
$$

where $\mathcal{L}_{\text {kin }}$ contains the kinetic terms for all gauge fields, quarks and leptons. $\psi_{L}^{T}=\left(t_{L}, b_{L}\right)$ is the third generation doublet of quarks containing the left-handed top- and bottom-fields and $t_{R}$ is the right-handed component of the top-quark. Due to the non-renormalizable structure of the model it is necessary to introduce a high energy cutoff $\Lambda$. The model can then be studied in the large $N_{c}$ limit (where $N_{c}$ is the number of colours). The so derived gap equation

$$
m_{t}=\frac{1}{2} G<\bar{t} t>=\frac{N_{c} G m_{t}}{8 \pi^{4}} \int_{0}^{\Lambda} d^{4} k \frac{i}{k^{2}-m_{t}^{2}}
$$

is found to be critical for $G>G_{c r}=8 \pi^{2} / N_{c} \Lambda^{2}$ and a top-condensate emerges which leads to a top-mass $m_{t}=\frac{1}{2} G<\bar{t} t>$. A typical feature of top-condensation is the separation of the cutoff scale $\Lambda$ from the top-mass scale. This is achieved by a fine-tuning of $G$ towards $G_{c r}$.

The separation of scales allows a more elegant approach to calculate the predictions of topcondensation, the so called renormalization group approach. To describe this concept we use the auxiliary field formalism where the four-Fermion coupling $G$ is intermediated by a non-propagating scalar doublet $\varphi$ of mass $G^{-1}$. This leads to the Lagrangian

$$
\mathcal{L}=\mathcal{L}_{\text {kinetic }}-\bar{\psi}_{L} \varphi t_{R}-\bar{t}_{R} \varphi^{\dagger} \psi_{L}-G^{-1} \varphi^{\dagger} \varphi
$$

At low energies a propagating Higgs field should emerge as a top-antitop-boundstate. This means that the Lagrangian of the standard model can be seen as the low energy effective Lagrangian of the top-condensation model. The standard model Lagrangian

$$
\mathcal{L}_{S M}=\mathcal{L}_{\text {kinetic }}+\left(D_{\mu} \phi\right)^{\dagger}\left(D^{\mu} \phi\right)-\frac{\lambda}{2}\left(\phi^{\dagger} \phi\right)^{2}+m^{2} \phi^{\dagger} \phi-g_{t}\left(\bar{\psi}_{L} \phi t_{R}+\bar{t}_{R} \phi^{\dagger} \psi_{L}\right)
$$

\footnotetext{
${ }^{2}$ We don't write colour indices explicitly
} 
can be rewritten using $\varphi:=g_{t} \phi$ for a better comparison with eq. (3):

$$
\mathcal{L}=\mathcal{L}_{\text {kinetic }}+\frac{1}{g_{t}^{2}}\left(D_{\mu} \varphi\right)^{\dagger}\left(D^{\mu} \varphi\right)-\frac{\lambda}{2 g_{t}^{4}}\left(\varphi^{\dagger} \varphi\right)^{2}+\frac{m^{2}}{g_{t}^{2}} \varphi^{\dagger} \varphi-\left(\bar{\psi}_{L} \varphi t_{R}+\bar{t}_{R} \varphi^{\dagger} \psi_{L}\right)
$$

Now we have to require that eq. (5) becomes eq. (3) at a certain scale $\Lambda$. This leads to the following conditions:

$$
\lim _{\mu^{2} \rightarrow \Lambda^{2}} g_{t}^{-2}\left(\mu^{2}\right)=0, \quad \lim _{\mu^{2} \rightarrow \Lambda^{2}} \frac{\lambda\left(\mu^{2}\right)}{g_{t}^{4}\left(\mu^{2}\right)}=0, \quad \lim _{\mu^{2} \rightarrow \Lambda^{2}} \frac{m^{2}\left(\mu^{2}\right)}{g_{t}^{2}\left(\mu^{2}\right)}=-G^{-1},
$$

where $\Lambda$ is the high energy cutoff of the top-condensation model. In underlying theories this cutoff corresponds to the mass of the heavy interaction particles.

It is important to notice that the conditions of eq. (6) have to obey both, renormalization group running and temperature-like quadratic running 1 . The goal is to connect the low energy standard model with the tree level top-condensation Lagrangian at the cutoff scale. First we reach the standard model at the cutoff scale using the renormalization group running of the standard model parameters. But this is not the whole story. The standard model is the effective theory of top-condensation with all its cutoff regularized loop contributions. These corrections are responsible for the symmetry breaking structure of the standard model scalar potential. To get the top-condensation tree level Lagrangian, it is necessary to subtract the cutoff regularized loop contributions. However we describe the situation in the framework of the effective theory, thus we need something which mirrors these subtractions in the effective theory. The quadratic running of the Higgs mass parameter $m^{2}=\lambda v^{2}$ does this job.

To see this explicitly we have a closer look at the top-condensation mechanism at the level of loop summation in the large $N_{c}$-limit: We start off with an effective top-condensation model with the Lagrangian eq. (11). Now we write down the 4-fermion interaction term including corrections in the large $N_{c}$-limit which comes up to the infinite bubble sum shown in fig. 11.

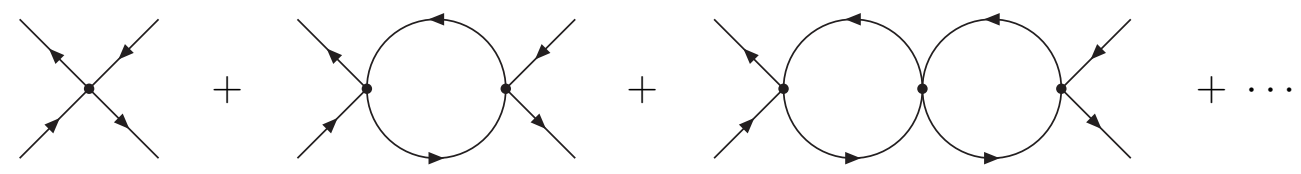

Figure 1 Bubble sum for the (pseudo)scalar boundstate propagator

This summation leads to an amplitude

$$
\Gamma\left(p^{2}\right)=\frac{1 / N_{c}}{1 / G-I}
$$

\footnotetext{
${ }^{3}$ The name RG-approach therefore is a little bit misleading. We nevertheless use this name as the name under which the concept was introduced.
} 
where $p$ is the outer momentum of the quarks and $G^{2} I$ is the integral over one bubble up to a cutoff $\Lambda$. There are four different amplitudes of the type eq. (7). A scalar and a pseudoscalar for top-top and positively and negatively charged pseudoscalar top-bottom amplitudes. To see the principle we just look at the scalar top-top amplitude. We get

$$
I_{s 4}\left(p^{2}\right)=\frac{-i}{2} \operatorname{tr} \int \frac{d^{4} k}{(2 \pi)^{4}} \frac{\left(\not k+m_{t}\right)\left(\not k-\not p+m_{t}\right)}{\left(k^{2}-m_{t}^{2}\right)\left[(k-p)^{2}-m_{t}^{2}\right)}
$$

which can be written as

$$
I_{s 4}\left(p^{2}\right)=\underbrace{\frac{1}{8 \pi^{4}} \int d^{4} k \frac{i}{k^{2}-m_{t}^{2}}}_{I_{1}}-\underbrace{\frac{\left(p^{2}-4 m_{t}^{2}\right)}{8 \pi^{4}} \int d^{4} k \frac{i}{\left(k^{2}-m_{t}^{2}\right)\left[(k-p)^{2}-m_{t}^{2}\right]}}_{I_{2}}
$$

where we have separated a quadratically divergent term $I_{1}$ and a logarithmically divergent term $I_{2}$. However $I_{1}$ is exactly the tadpole of the gap equation eq. (2) so that $1-G I_{1}=0$. Inserting this into eq. (7) we get an amplitude

$$
\Gamma_{s 4}\left(p^{2}\right)=\frac{1}{N_{c} I_{2}}=\frac{1}{\left(p^{2}-4 m_{t}^{2}\right) N_{c} \frac{1}{(4 \pi)^{2}} \ln \frac{M^{2}}{p^{2}}+\text { finite terms }}
$$

where all large couplings $G$ have fallen out. The use of the finetuned gap-equation led to a scalar propagator with a small mass $M_{H}=2 m_{t}$. This scalar top-antitop boundstate shows up as an effective Higgs field in the low energy Lagrangian.f

Now we introduce an infrared cutoff $\mu$ in the loop integrals and shift that cutoff continuously up towards $\Lambda$. By doing so we reduce the loop contributions until we finally get back to tree-level top-condensation for $\mu=\Lambda$. After shifting $\mu$ above the electro-weak scale we loose the relation $1-G I_{1}=0$ and therefore the finetuning. Thus we can understand eq. (9) approximately as a heavy scalar propagator of mass $\mu$. At $\mu=\Lambda$ all loop contributions have vanished and we end up with a nonpropagating auxiliary Higgs like it was introduced in eq. (3). If we write our Higgs propagator into the effective Lagrangian, we see explicitly now, that a continuous subtraction of the loop contributions appears there as a quadratic running of the scalar mass term. This makes it possible to meet the third condition of eq. (6).

\footnotetext{
${ }^{4}$ The mass relation $M_{H}=2 m_{t}$ looks very intuitive for a top-antitop boundstate but is only valid in this simple approximation. The RG-approach gives a lower value.

${ }^{5}$ the pseudoscalar top-top and top-bottom amplitudes lead to the massless Goldstone bosons following the same arguments.

${ }^{6}$ For the sake of simplicity we have developed our argument in the framework of simple loop summation. To get an exact matching between the logarithmic contributions of the dynamical calculations and the RGcalculations, it would be necessary to use an improved approach involving a running top mass function. (See [5]) However, the simpler approach is sufficient to understand the role of the quadratic running.
} 
The fulfillment of the three conditions by the described running implies certain values for the top- and the Higgs mass depending on the VEV (fixed by the W-mass) and the scale of the Landau pole of the top-Yukawa coupling (fixed by the cutoff scale). The fulfillment of the pole conditions by RG-running compared with different standard model scenarios (fig. 2) is shown in fig. 3 for a cutoff scale of $10^{4} \mathrm{GeV}$.

These conditions give a too high top-mass prediction in the simplest model. However one can construct extensions by introducing additional four-Fermion couplings [14], enlarging the gauge group [15] or supersymmetrizing the theory [16] to achieve a phenomenologically viable value.

Up to now we have described the RG-approach in the framework of minimal topcondensation. Now we want to ask what are the general conditions which make a RGdescription of a theory of dynamical symmetry breaking possible.

Two preconditions have to be met: First the scale of the interaction responsible for dynamical symmetry breaking must be considerably higher than the mass scale of the bound states. If this separation of scales does not exist the region where a $R G$-description could apply is zero. Second the light or massless fields of the full theory must also appear in the low energy theory. This is necessary to make an identification of parameters of the effective and the

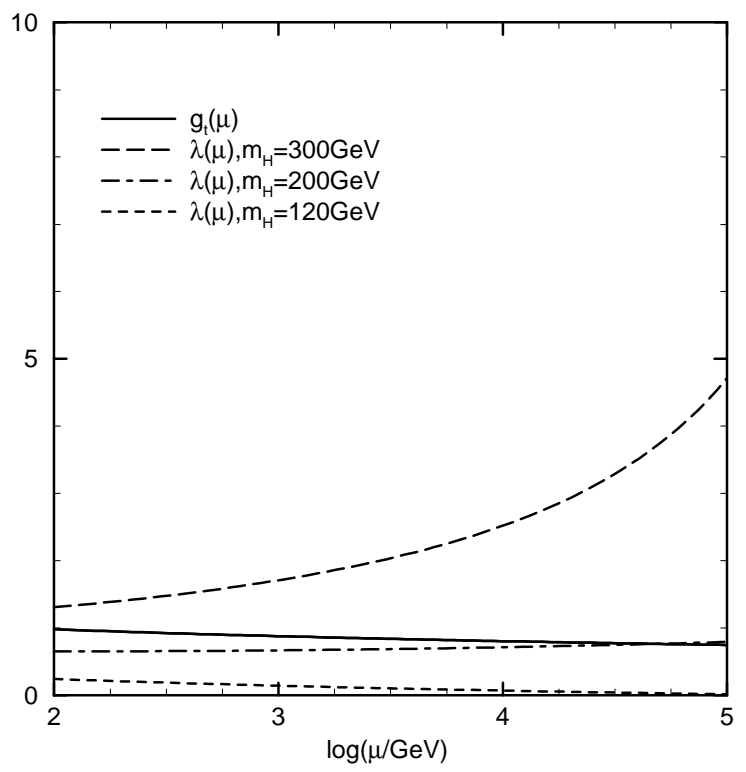

Figure $2 R G$-evolution in the standard model for $\left(m_{t}\right)_{\overline{M S}}=167 \mathrm{GeV}$.

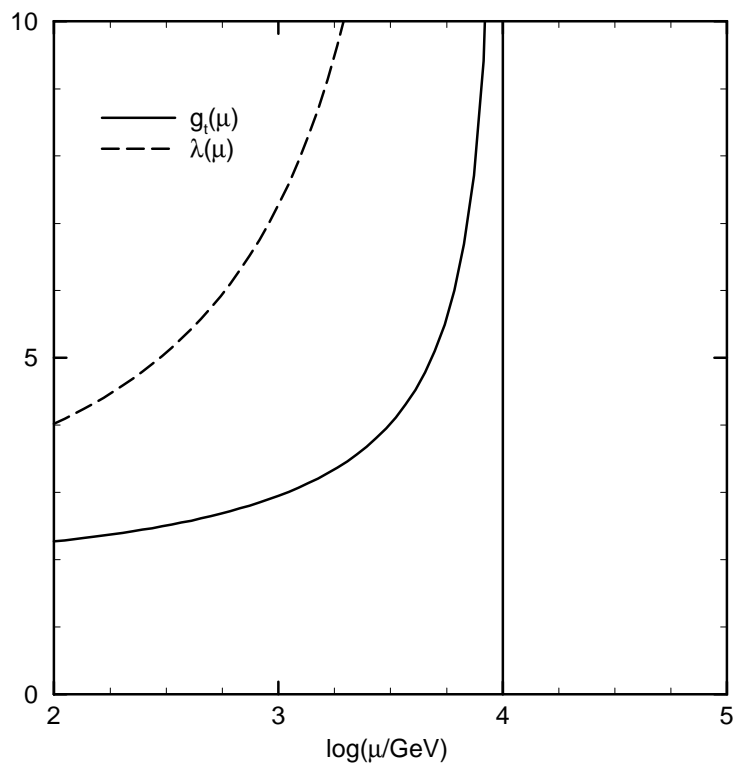

Figure $3 R G$-evolution in the simple top-condensation model for a cutoff scale $\Lambda=10^{4} \mathrm{GeV}$. 
full theory possible. The first condition is not fulfilled in technicolour models where the new interaction scale is not separated from mass scale of the techniquarks. The second condition is neither fulfilled in technicolour nor in preonic models because techniquarks and preons do not appear in the low energy theory due to confinement. If however both conditions are fulfilled, as it is the case in top-condensation, a $\mathrm{RG}$-formulation always exists and is not devaluated by higher dimensional operators. One can always formulate a low energy effective theory which includes the whole dynamics of the model. From this low energy effective theory one must get back to the tree structure of the full theory if one considers RG-running up to the critical scale and subtracts all loop corrections. We have seen in the minimal case that this subtraction corresponds to considering quadratic running of the scalar mass parameter in the effective theory. This situation is not changed at all by higher dimensional operators. The higher dimensional operators just change the identification conditions for the effective theory which are based on renormalization group running [13].

In the forthcoming sections we will use this extension of the RG-approach to learn about models with higher dimensional operators. Our basic idea will be the following: We will assume that a RG-formulation of the discussed model exists. Then we investigate what the implications of the mere existence of such a formulation are. It will turn out that

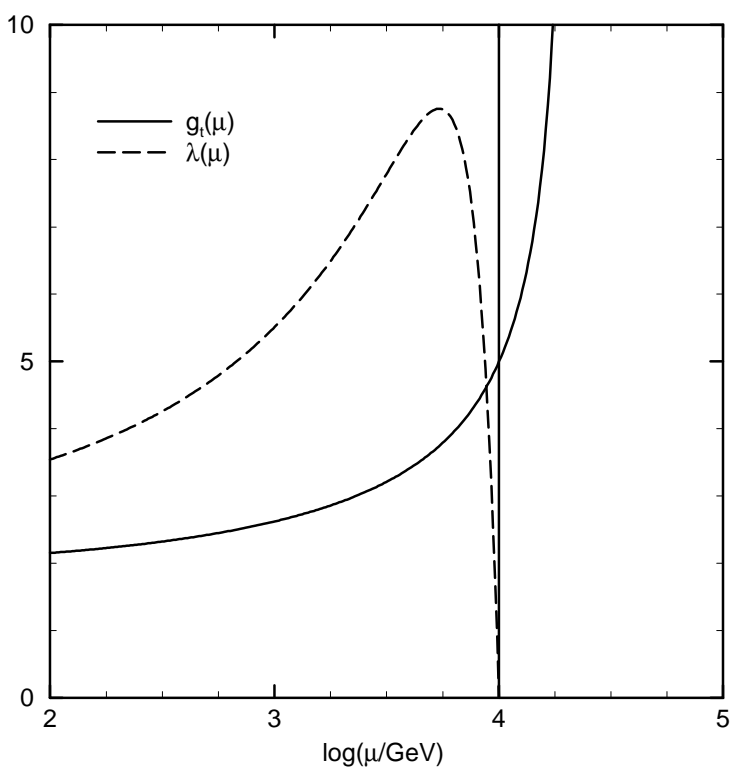

Figure $4 R G$-evolution for the case of heavy scalar exchange.

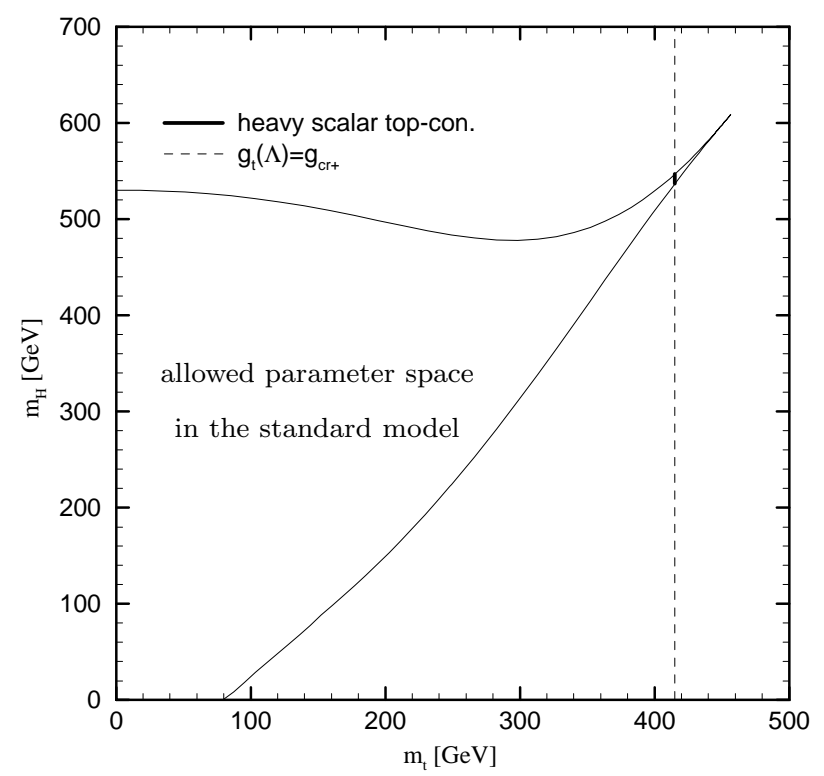

Figure 5 Allowed parameter space in the standard model and in "heavy scalar mediated top-condensation" for $\Lambda=10^{4} \mathrm{GeV}$. 
these implications are by far stronger than one would expect. For sake of simplicity we do our discussions in the simplest model. The conclusions apply to all non-supersymmetric generalizations \.

\section{$3 \quad$ Higher Dimensional Operators}

Soon after the invention of top-condensation Hasenfratz et al. [7] and Zinn-Justin [8] showed that top-condensation with a specific set of higher dimensional operators represents a reparametrization of the standard model. We will reproduce their arguments in the framework of the extended RG-approach which gives a very intuitive picture. We consider a low energy standard model scenario with arbitrary top- and Higgs mass. Now we just follow the procedure described at the end of the last section. We choose an arbitrary cutoff scale and ask, whether it is possible to connect the low energy standard model Lagrangian with a general top-condensation Lagrangian at this cutoff scale. As it will be shown in the following the answer to this question is yes. The renormalization group running in general will not lead to a Landau pole at the cutoff scale, we will still face scalar kinetic terms there. The quadratic running of the Higgs mass parameter will give a positive mass term of the order $\Lambda$ just like in standard top-condensation. To match the two Lagrangians we therefore have to find a correspondence to the kinetic terms of the standard model Higgs field in the top-condensation Lagrangian. We start with the low energy Lagrangian eq. (4) neglecting the gauge couplings for simplicity. We consider renormalization group running up to the cutoff scale $\Lambda$ which leads to the Lagrangian

$$
\mathcal{L}_{S M}(\Lambda)=\mathcal{L}_{k i n}(\Lambda)+\left(\partial_{\mu} \phi\right)^{\dagger}\left(\partial^{\mu} \phi\right)-\frac{\lambda(\Lambda)}{2}\left(\phi^{\dagger} \phi\right)^{2}+m^{2}(\Lambda) \phi^{\dagger} \phi-g_{t}(\Lambda)\left(\bar{\psi}_{L} \phi t_{R}+\bar{t}_{R} \phi^{\dagger} \psi_{L}\right)
$$

We consider quadratic running (qr) of the scalar mass term which leads to

$\mathcal{L}_{q r}(\Lambda)=\mathcal{L}_{k i n}(\Lambda)+\left(\partial_{\mu} \phi\right)^{\dagger}\left(\partial^{\mu} \phi\right)-\frac{\lambda(\Lambda)}{2}\left(\phi^{\dagger} \phi\right)^{2}-\frac{g_{t}^{2}(\Lambda)}{g_{c r_{+}}^{2}} \Lambda^{2} \phi^{\dagger} \phi-g_{t}(\Lambda)\left(\bar{\psi}_{L} \phi t_{R}+\bar{t}_{R} \phi^{\dagger} \psi_{L}\right)$

where we defined a dimensionless coupling $g_{c r_{+}}$by $G:=\frac{g_{c r_{+}}^{2}}{\Lambda^{2}}$. In a fine-tuned model $(G \cong$ $\left.G_{c r}\right) g_{c r_{+}}$is only slightly above the critical value $g_{c r}$, the corrections are of the order $g_{c r_{+}}=$ $g_{c r}+O\left(\frac{\mu_{e l w}^{2}}{\Lambda^{2}} \ln \frac{\Lambda}{\mu_{e l w}}\right)$. We can see that, as we keep the terms $\left(\partial_{\mu} \phi\right)^{\dagger}\left(\partial^{\mu} \phi\right)$ at the cutoff scale, the redefinition $\varphi:=g_{t} \phi$ is not infinite. However, to get a clear picture of what is going on,

\footnotetext{
${ }^{7}$ In a RG-treatment of supersymmetric top-condensation quadratic running plays no role due to the cancellations of quadratic divergencies. Therefore the arguments of our discussion are not valid.
} 
we choose a redefinition $\varphi:=\frac{g_{t}(\Lambda)}{g_{c r_{+}}} \phi$. We get

$\mathcal{L}_{q r}(\Lambda)=\mathcal{L}_{k i n}(\Lambda)+\frac{g_{c r_{+}}^{2}}{g_{t}^{2}(\Lambda)}\left(\partial_{\mu} \varphi\right)^{\dagger}\left(\partial^{\mu} \varphi\right)-\frac{\lambda(\Lambda) g_{c r_{+}}^{4}}{2 g_{t}^{4}(\Lambda)}\left(\varphi^{\dagger} \varphi\right)^{2}-\Lambda^{2} \varphi^{\dagger} \varphi-g_{c r_{+}}\left(\bar{\psi}_{L} \varphi t_{R}+\bar{t}_{R} \varphi^{\dagger} \psi_{L}\right)$

We have separated the cutoff suppression scale from the Yukawa coupling of the auxiliary field. The heavy mass term represents purely the compensation of the cutoff contribution while the Yukawa coupling controls dynamical symmetry breaking and is fine-tuned against the coupling where the perturbation expansion breaks down. We are very close to a scenario with heavy scalar exchange of mass $\Lambda$ in a strong coupling regime. The only deviation is the factor $\frac{g_{r_{+}}^{2}}{g_{t}^{2}(\Lambda)}$ in front of the scalar kinetic term. Of course one could find a different normalization for $\varphi$ where this factor is one, but in this case we would loose the connection between the heavy mass and the cutoff scale as well as the right size for the Yukawa coupling which has to be strong to induce dynamical symmetry breaking, if we take the interpretation of our Lagrangian as a description of heavy particle exchange seriously. What we have constructed is therefore not heavy scalar exchange but just a top-condensation model with higher dimensional operators in auxiliary field formalism.

We expand now in the heavy scale $\Lambda$ by inserting the Euler-Lagrange equations for $\varphi$ and $\varphi^{\dagger}$ repeatedly to get a series of higher dimensional operators. The Euler Lagrange equations are

$$
\begin{aligned}
\varphi & =\frac{1}{\Lambda^{2}}\left[-g_{c r_{+}} \bar{t}_{R} \psi_{L}-\frac{g_{c r_{+}}^{2}}{g_{t}^{2}(\Lambda)} \partial^{2} \varphi-\frac{g_{c r_{+}}^{4} \lambda(\Lambda)}{g_{t}^{4}(\Lambda)} \varphi \varphi^{\dagger} \varphi\right] \\
\varphi^{\dagger} & =\frac{1}{\Lambda^{2}}\left[-g_{c r_{+}} \bar{\psi}_{L} t_{R}-\frac{g_{c r_{+}}^{2}}{g_{t}^{2}(\Lambda)} \partial^{2} \varphi^{\dagger}-\frac{g_{c r_{+}}^{4} \lambda(\Lambda)}{g_{t}^{4}(\Lambda)} \varphi^{\dagger} \varphi \varphi^{\dagger}\right]
\end{aligned}
$$

which leads to the following expansion of $\varphi$ and $\varphi^{\dagger}$ :

$$
\begin{gathered}
\varphi=-\frac{g_{c r_{+}}}{\Lambda^{2}} \bar{t}_{R} \psi_{L}+\frac{g_{c r_{+}}}{\Lambda^{4}} \partial^{2}\left(\bar{\psi}_{L} t_{R}\right)+O\left(\Lambda^{-6}\right), \\
\varphi^{\dagger}=-\frac{g_{c r_{+}}}{\Lambda^{2}} \bar{\psi}_{L} t_{R}+\frac{g_{c r_{+}}}{\Lambda^{4}} \partial^{2}\left(\bar{t}_{R} \psi_{L}\right)+O\left(\Lambda^{-6}\right) .
\end{gathered}
$$

Reinserting this expansion into eq. (13) leads to the new top-condensation Lagrangian:

$$
\mathcal{L}_{t c}(\Lambda)=\frac{g_{c r_{+}}^{2}}{\Lambda^{2}} \bar{\psi}_{L} t_{R} \bar{t}_{R} \psi-\frac{g_{c r_{+}}^{2}}{\Lambda^{4}}\left(\partial_{\mu}\left(\bar{t}_{R} \psi_{L}\right)\right)^{\dagger}\left(\partial_{\mu}\left(\bar{t}_{R} \psi_{L}\right)\right)+O\left(\Lambda^{-6}\right)
$$

The resulting higher dimensional operators are those suggested by Hasenfratz et al. This discussion tells us that every standard model Lagrangian can be understood as the low 
energy effective theory of a top-condensation model with suitable higher dimensional operators. There exists a specific set of higher dimensional operators for any choice of the top condensation cutoff scale below eventual Landau poles.

This does not automatically mean that top-condensation models including such sets of higher dimensional operators are meaningless. If some couplings become non-local at the cutoff scale, the top-condensation could still be distinguished from the standard model. It would be a reasonable theory in its own right, though not easily detectable at low energies. If however such nonlocality does not appear at the cutoff scale, the higher dimensional operators just describe the propagation of the Higgs at this scale. In this case the standard model is not just a low energy effective theory of top-condensation up to the cutoff but top-condensation and the standard model are identical at all scales. They are just different parametrizations of the same model.

\section{Relevant and Irrelevant Operators}

In order to get a clear understanding which higher dimensional operators turn a specific model of top-condensation into a new theory and which ones keep it a pure reparametrization of the standard model we have to introduce the notations of relevant and irrelevant operators in the renormalization group framework. Relevant operators at the electro-weak scale are those operators which contribute there without a high suppression scale. Usually all operators stemming from the exchange of heavy particles or from non-renormalizable interaction at a high scale will be irrelevant at low scales. This is however not always the case. Especially in top-condensation, being a fine-tuned theory, the relevance of higher dimensional operators suppressed by the cutoff scale is a basic principle of the theory. For example the four-Fermion coupling term

$$
O_{4}=G \bar{\psi}_{L} t_{R} \bar{t}_{R} \psi_{L}
$$

is suppressed by the heavy scale. However if it would not be relevant at the electro-weak scale, we could not see any light electro-weak breaking phenomenology induced by topcondensation. The relevance of this operator can be seen in the framework of the RGapproach. In auxiliary fields eq. (19) appears as a heavy mass term

$$
O_{4 H}=G^{-1} H^{\dagger} H
$$

where $H=G \bar{\psi}_{L} t_{R}$. Now the quadratic running connects this term to the small Higgs mass parameter of the standard model which obviously is relevant at low scales. We see that 
the fine-tuning of the theory has made highly suppressed operators relevant at low scales, which is formally described by the quadratic running of the scalar mass parameter from a high to a low value. There is no reason why the simple four-Fermion coupling of eq. (19) should be the only operator in a top-condensation model that is relevant at the electro-weak scale. We have seen other such operators already in section 3, where the higher dimensional operators obviously change the low energy phenomenology considerably and therefore must be relevant. On the other side not every suppressed operator in a top-condensation model is relevant at low scales. Our goal is now to find a way to distinguish between relevant and irrelevant operators.

It is possible to give a simple general definition of a relevant operator in the framework of the renormalization group approach. We start with some model at a high scale $\mu_{h}$ consisting of a number of mass terms, dimension-four operators and higher dimensional operators. This model must have an effective theory at a lower scale $\mu_{l}$ in which all higher dimensional operators suppressed by $\mu_{h}$ are neglected. The renormalization group approach connects the low energy effective theory with the full theory at $\mu_{h}$ by $\mathrm{RG}-$ and quadratic running. In general, however, not all operators of the full theory can be identified with operators of the effective theory. Those which can be identified are low energy relevant operators as they contribute to the low energy effective theory in form of the identifiable effective operators. Those which cannot be identified are irrelevant. They occur at low energies only in form of operators suppressed by the high scale and can be neglected.

Using the notation of irrelevant operators it is possible to give a clear definition of what is a mere reparametrization of the standard model and what is a theory in its own right:

A theory that is distinguishable from the standard model must have irrelevant operators at the high scale. These irrelevant operators are not part of the effective theory and therefore distinguish this effective theory from the full theory at higher scales. A theory which does not have irrelevant operators is fully described by the "effective" theory and therefore not more than its reparametrization. We will see examples for both cases in the upcoming sections. The simplest example for a mere reparametrization is minimal top-condensation without any underlying concept, where all high scale operators are relevant at low energies.8 To give top-condensation a physical meaning it is necessary to introduce an underlying concept that contains irrelevant operators. The next section will discuss that heavy scalar exchange is

8 This case is a little bit special however. While the standard model cannot be defined above the Landau pole top-condensation formally could due to the infinite field redefinition in course of the RGapproach. However this cannot be more than a formal continuation since the notation of a dimensionful non-renormalizable coupling is not physically senseful above that coupling scale. 
no viable possibility since it is a standard model reparametrization itself. An example for a true underlying theory in its own right is heavy vector exchange which we will discuss in section 7 .

\section{Heavy Scalar Exchange}

Our method in section 3 was to construct exactly the set of higher dimensional operators which can be connected with a specific standard model Lagrangian through RG- plus quadratic running. Due to the discussion in section 4 this means that we do not have any irrelevant operators in our Lagrangians. Therefore we constructed in fact pure reparametrizations of the standard model.

The higher dimensional operators of section 3 however look very similar to those produced by heavy scalar exchange. Actually they are identical with heavy scalar exchange for the choice $g_{t}(\Lambda)=g_{c r_{+}}$. In this case the factor in front of the scalar kinetic term of eq. (13) is one and we get pure heavy scalar mediated top-condensation. Heavy scalar exchange thus does not produce any irrelevant operators, it is not a theory in its own right but just a special case of the standard model reparametrizations constructed in section 3. It is a reparametrization of a standard model with a Yukawa coupling $g_{t}$ that becomes strong at the mass scale of the heavy scalar.

There have been investigations of scalar mediated top-condensation in [10] where the results differ from the standard model case. We will just argue shortly why these results are incorrect according to our understanding.

[10] uses the standard model scalar sector, however with a positive heavy mass term in the scalar potential:

$$
V(\Phi)=m_{\phi}^{2}|\Phi|^{2}+\lambda|\Phi|^{4}
$$

Only the s-channel scalar exchange diagrams contribute in lowest order $1 / N_{c}$. For a sufficiently large top-Yukawa coupling top-condensation can occur. The authors observe that the top-condensate gives an additional contribution to the scalar potential which becomes

$$
V(\Phi)=m_{\Phi}^{2}|\Phi|^{2}+\lambda|\Phi|^{4}+\frac{g_{t}}{\sqrt{2}}<\bar{t} t>\phi_{3}
$$

and produces a final VEV of $\phi_{3}$ which is

$$
<\phi_{3}>=v=-\frac{g_{t}<\bar{t} t>}{\sqrt{2} m_{\phi}^{2}} .
$$


This is interpreted as a sign for a light component of the fundamental scalar field in addition to the composite Higgs field. However this is not correct. The misinterpretation is based on a misunderstanding of the role of the quadratic contributions in top-condensation. As discussed in section 2 these connect the tree level top-condensation Lagrangian with its effective low energy Lagrangian. Therefore the light scalar component found above after considering quadratic loop contributions cannot be interpreted in the framework of topcondensation, it has to be understood in the framework of the effective theory. With this knowledge the message of eq. (23) is very clear. The standard model scalar can be identified with the heavy scalar of top-condensation corrected by its quadratic contributions. What is found in [10] is not an additional scalar but nothing else than the standard model Higgs.

Figures 2-5 illustrate the three scenarios discussed so far. Fig. 2 describes the RG-running of the top Yukawa coupling and the four Higgs coupling in the standard model case with realistic parameters. It would be possible to use any scale below the Landau pole of the Higgs quartic coupling as a cutoff scale in an extended top-condensation scenario as described in section 3 . Fig. 3 describes simple top-condensation without higher dimensional operators. Fig. 4 shows one case of heavy scalar exchange $(\lambda(\Lambda)=0)$ which however itself is just a special case of a standard model reparametrization. One can see that the heavy scalar exchange for arbitrary four-scalar coupling $\lambda_{S}$ of the heavy scalar always remains very close to the minimal topcondensation case. The deviations of the low energy parameters from the minimal case are a few percent. This is a consequence of the so called quasi infrared fixed point behaviour of the $\mathrm{RG}$-running that forces the parameters into a small region of low energy values if they run down from Landau poles at considerably varying high scales. Nevertheless the operators responsible for the difference between minimal and scalar intermediated top-condensation of course must be classified as relevant operators for low energy physics. While the quasi fixed point for a high scale around $10^{15} \mathrm{GeV}$ suppresses the importance of high energy variations to a few percent, irrelevant operator according to our definition would be suppressed by $\mu_{h} / \mu_{l} \sim 10^{-13}$.

Fig. 5 [17] shows the segment of the standard model parameter space which can be reparametrized by "heavy scalar mediated top-condensation". It is defined by the intersection of the allowed parameter region for the standard model case and the $m_{t}$ line which leads to the condition $g_{t}(\Lambda)=g_{c r_{+}}$. The Higgs mass is not fixed exactly in this scenario, it depends on the value of the four-scalar coupling of the heavy scalars. However due to the infrared fixed point behaviour the region of allowed Higgs masses, restricted by the pole for $\lambda$ at the cutoff from above and by $\lambda=0$ at the cutoff from below is very small. The point in parameter space corresponding to standard top-condensation would be the intersection 
between vacuum stability and triviality bound (the lower and upper bound of the allowed parameter space for the standard model).

A last point which should be addressed is the t-channel scalar exchange. Because of the $1 / N_{c}$-expansion it is only the dominant case for a colour octet scalar interaction which is repulsive.

\section{Composite Vectors}

One open question in top-condensation has been the behaviour of vector bound states. In the simplest top-condensation model they do not appear due to the specific structure of the four-Fermion coupling $G$. However any natural extension of $G$, especially any effective coupling stemming from heavy vector exchange, will include operators of the type

$$
G^{\prime}\left(\bar{\psi}_{L} \gamma_{\mu} \psi_{L}\right)\left(\bar{\psi}_{L} \gamma^{\mu} \psi_{L}\right)+G^{\prime \prime}\left(\bar{t}_{R} \gamma_{\mu} t_{R}\right)\left(\bar{t}_{R} \gamma^{\mu} t_{R}\right)
$$

and may consequently produce vector boundstates. The question is, on which scale these boundstates will appear in a fine-tuned model. Will they be lowered like the scalar boundstates, will they remain at the heavy scale or will they be somewhere in between? Most of the investigations about vector bound states in NJL-like models were done in the context of QCD (see for example [12] and references therein) which is no fine-tuned model. The question of scale for the vector bound states therefore does not arise in that case. In a top-condensation framework just the role of hypothetical vector boundstates at arbitrary scales was investigated in [11]. The actual scale of these fields remained unknown.

It turns out that the extended $\mathrm{RG}$-approach gives an answer to this question. If we imagine a vectorial boundstate with a mass considerably lower than the cutoff scale, then the RGapproach demands the identification of the corresponding operators of the effective theory with operators of the top-condensation theory at the cutoff scale. Since all top-condensation operators are connected to the cutoff scale the expansion in the heavy scale starts with dimension six operators:

$$
\mathcal{L}_{t c}=\frac{1}{\Lambda^{2}} O(6)+\frac{1}{\Lambda^{4}} O(8)+\ldots
$$

Therefore the vectorial mass must be identified with a cutoff scale mass term. Due to the lower mass scale of the vector state this identification cannot be provided by RG-running. It must be provided by quadratic running. Now our theory is a theory of dynamically broken gauge symmetry. The gap-equation produces a condensate which breaks the symmetry and 
serves as a mass term for the light fields. The low scale of those masses is achieved by a fine-tuning of the effective coupling $G$ towards the critical coupling of the gap equation. Therefore any low mass term must be produced by the gap equation, in other words any field which gets a low scale mass has to couple to the condensate. In the effective theory the top-condensate corresponds to the VEV of the Higgs field. Thus if vector boundstates would acquire fine-tuned masses from the gap equation, these would have to be produced by coupling the fields to a Higgs VEV:

$$
\mathcal{L}_{V}=(\partial V)^{2}+V^{2}<v>^{2}+\bar{\psi} \gamma^{\mu} \psi V_{\mu}
$$

Of course fundamental mass terms for vector-boundstates can exist, those are however not induced by the condensate but directly by the heavy interaction scale. They have consequently no connection to the fine-tuning of the theory and therefore necessarily remain at the high scale. The only possible low scale quadratic mass terms in the effective theory are the mass parameters of the scalar potential that will finally produce the VEV. These mass parameters however change their sign in course of their quadratic running at the breaking scale $\mu_{b r}<\Lambda$, which puts the VEV to zero. Consequently all vector mass terms vanish at $\mu_{b r}$ if we consider quadratic running.

$$
\mathcal{L}_{V}^{q r}\left(\mu_{b r}\right)=(\partial V)^{2}+\bar{\psi} \gamma^{\mu} \psi V_{\mu}
$$

It is therefore not possible for a vector mass term to run up to a high scale by quadratic running. Thus one cannot connect low vectorial mass terms with high scale operators of top-condensation. All low scale vectorial mass terms remain low mass terms in the topcondensation theory, none of them can possibly be interpreted as a dynamically produced boundstate. All vector boundstates in a top-condensation theory must have a mass of the order cutoff scale. $]^{9}$

\section{Heavy Vector Exchange}

Heavy vector exchange is the standard concept to introduce an underlying theory for the nonrenormalizable four-Fermion interaction. Of course this concept implies additional higher dimensional operators at the cutoff scale. Now once again there arises the question whether

\footnotetext{
${ }^{9}$ The statement that vector-boundstates cannot be lowered by fine-tuning seems to be rather general. For example it remains true for a Nambu-Jona-Lasinio model without dynamical gauge symmetry breaking. The breakdown of the chiral symmetry due to the Fermion condensate is sufficient to keep our argument valid.
} 
these operators can change the low energy prediction of top-condensation. This question was addressed in [13] where box diagrams of the type

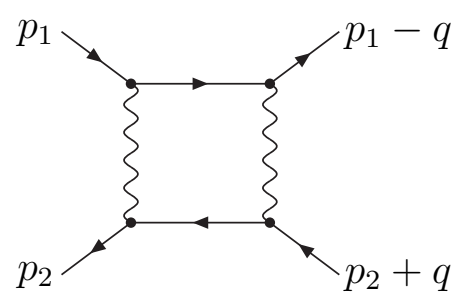

are studied for a special example (a specific gauge structure). The conclusion was that the scalar kinetic contributions produced by these diagrams are about 100 times smaller than it would be necessary to give a considerable effect. This was however just shown for one example and restricted to box diagrams. The statement that the situation will not change in other models or at higher orders basically remained a plausibility argument. We use the arguments developed in the last sections to get a more substantial understanding of this question.

The crucial point of this argument has already been stated in section 2. The RG-approach connects the tree level standard model Lagrangian (which is the effective formulation of the top-condensation model with all corrections) to the tree-level top-condensation Lagrangian at the cutoff scale. We once more want to emphasize that this is not just the case in the minimal model. It is a necessary feature of all extensions as well, rooted in the nature of finetuned dynamical symmetry breaking: There exist highly suppressed tree level operators (in effective top-condensation four fermion coupling terms, in a full model e.g. strongly coupled heavy vector exchange), which nevertheless produce low energy relevant effective operators via their dynamics (loop contributions). The mechanism of the RG-approach connects these low energy relevant tree operators to their low energy remnants, the operators of the standard model. As the whole low energy phenomenology stems from the dynamics induced by the tree level top-condensation Lagrangian, in the RG-framework one can say everything about the low energy theory by connecting it to this tree level Lagrangian. The fact that we connect to tree level operators is reflected in the quadratic running of the scalar mass term which represents the subtraction of the loop amplitudes enhanced by finetuning.

If we would connect the standard model to top-condensation including all loop corrections, we would have to use just the logarithmic RG-running without involving the quadratic running of the scalar mass parameter. This would come up to the impossible task of reproducing the low energy phenomenology by calculating the full dynamics of a strongly interacting gauge theory. 
We conclude

1. that it is methodically wrong to include the box diagrams into the $\mathrm{RG}$-approach and

2. that the tree level top-condensation Lagrangian is sufficient to make this RG-approach exact.

Therefore all we have to do is analyze, which relevant higher dimensional operators we can get from tree level heavy vector exchange. In other words, we want to know which of these higher dimensional operators can be identified with operators of the effective theory.

Schematically the expansion of tree level vector exchange in powers of $1 / M^{2}$ looks like

$$
\begin{aligned}
\mathcal{L} & =-g \bar{\psi} \gamma_{\mu} \psi V^{\mu}-\frac{1}{4} F_{\mu \nu} F^{\mu \nu}+\frac{M^{2}}{2} V_{\mu} V^{\mu} \\
& =-\frac{g^{2}}{2 M^{2}} j_{\mu} j^{\mu}-\frac{g^{2}}{4 M^{4}} J_{\mu \nu} J^{\mu \nu}+O\left(M^{-6}\right),
\end{aligned}
$$

with

$$
j_{\mu}=\bar{\psi} \gamma_{\mu} \psi, J_{\mu \nu}=\left(\partial_{\mu} j_{\nu}-\partial_{\nu} j_{\mu}\right)
$$

Now one can compare this expansion with the expansion of tree level scalar exchange. While the dimension four operator can be transformed into the corresponding scalar exchange operator by fierzing, this is not possible for the higher dimensional operators due to their derivative couplings. This means that tree level vector exchange does not give scalar kinetic contributions. Boxes and higher loops, which would give scalar kinetic contributions, do not contribute to the RG-analysis, as we have discussed above. Thus these scalar kinetic contributions are just threshold effects which do not contribute to low energy physics. The $\mathrm{RG}$-description of heavy vector exchange connects to exactly the same set of high scale operators as the RG-description of minimal top condensation. The two descriptions are therefore identical. The difference in low energy predictions between the two models cannot be formulated in the framework of the renormalizable effective theory and therefore is not relevant.

\section{Connections to Dynamical Methods}

In the previous sections the relevance of higher dimensional operators was investigated with respect to the $\mathrm{RG}$-approach. We found that there are crucial differences between an underlying scalar theory and a vector boson exchange. These differences also have to be visible on the level of Schwinger-Dyson equations and it is the aim of this section to clarify this issue. 
Even in lowest order $1 / N_{c}$ the full theory cannot be solved. Thus all we can do is to investigate whether the lowest order dynamical calculations of heavy scalar respectively vector exchange already show the properties observed in the $\mathrm{RG}$-discussion.

We start by introducing a higher dimensional operator in the effective four-Fermion vertex:

$$
G=G_{\kappa=0}\left(1+\kappa \frac{p^{2}}{M^{2}}\right)
$$

If we consider scalar exchange with $G_{\kappa=0}=g^{2} N_{c} / M^{2}$ that term is contained in the expansion of the scalar propagator:

$$
(-i g) \frac{i}{p^{2}-M^{2}}(-i g)=\frac{i G_{\kappa=0}}{N_{c}}\left(1+\frac{p^{2}}{M^{2}}\right)+O\left(\frac{p^{4}}{M^{4}}\right)
$$

Now the crucial point is that the corresponding gap equation

$$
1=\frac{2 G_{\kappa=0}}{(4 \pi)^{2}}\left(\Lambda^{2}-m^{2} \ln \frac{\Lambda^{2}}{m^{2}}\right)
$$

still involves the coupling $G_{\kappa=0}$ without the additional contribution since there is no momentum flux into the tadpole. The Higgs propagator can be found by summing up the 1PI graphs

$$
i D=\frac{-i G}{g_{t}^{2} N_{c}}+\frac{-i G}{g_{t}^{2} N_{c}}(i \Pi) \frac{-i G}{g_{t}^{2} N_{c}}+\cdots=\frac{\frac{-i G}{g_{t}^{2} N_{c}}}{1-\Pi \frac{G}{g_{t}^{2} N_{c}}}
$$

where

$$
\begin{aligned}
i \Pi & =\frac{i g_{t}^{2} N_{c}}{(4 \pi)^{2}}\left(2 \Lambda^{2}+\left(p^{2}-6 m^{2}\right) \ln \frac{\Lambda^{2}}{p^{2}}\right) \\
& =i \frac{g_{t}^{2} N_{c}}{G_{\kappa=0}}+\frac{i g_{t}^{2} N_{c}}{(4 \pi)^{2}}\left(p^{2}-4 m^{2}\right) \ln \frac{\Lambda^{2}}{p^{2}}
\end{aligned}
$$

by using the gap equation. We find

$$
\begin{aligned}
i D & =\frac{-i G}{g_{t}^{2} N_{c}}\left(1-\frac{G}{G_{\kappa=0}}-\frac{G}{(4 \pi)^{2}}\left(p^{2}-4 m^{2}\right) \ln \frac{\Lambda^{2}}{p^{2}}\right)^{-1} \\
& =i\left(\left(\kappa \frac{g_{t}^{2} N_{c}}{G M^{2}}+\frac{g_{t}^{2} N_{c}}{(4 \pi)^{2}} \ln \frac{\Lambda^{2}}{p^{2}}\right) p^{2}-\frac{g_{t}^{2} N_{c}}{(4 \pi)^{2}} 4 m^{2} \ln \frac{\Lambda^{2}}{p^{2}}\right)^{-1} .
\end{aligned}
$$

The zero of the Higgs self-energy and therefore the Landau pole is now shifted due to the different four-Fermion couplings in the propagator and the gap equation. If all higher order terms are included in eq. (32) and the identification $g^{2}=g_{t}^{2}$ is made, one ends up with:

$$
i D=i\left[\left(1+\frac{g_{t}^{2} N_{c}}{(4 \pi)^{2}} \ln \frac{\Lambda^{2}}{p^{2}}\right) p^{2}-\frac{g_{t}^{2} N_{c}}{(4 \pi)^{2}} 4 m^{2} \ln \frac{\Lambda^{2}}{p^{2}}\right]^{-1}
$$


The zero of the Higgs self-energy and therefore the pole of the top-Yukawa coupling $g_{t}$ is not at the cutoff scale since we started with a scalar theory and a well defined finite $g_{t}$ at the cutoff. The Higgs self-energy is one at this scale so that $g_{t}$ there matches the value $g$ of the underlying Lagrangian. The pole of $g_{t}$

$$
\Lambda_{\mathrm{pol}}=\Lambda e^{\frac{(4 \pi)^{2}}{2 g_{t}^{2} N_{c}}}
$$

is beyond the cutoff. Since we did not introduce a heavy quartic Higgs coupling in our example, $\lambda$ must be zero at $\Lambda$. In the effective picture this is fulfilled by the Higgs mass in the propagator eq. (39).

In the case of a heavy vector exchange the situation changes drastically. In the $1 / N_{c}$ approximation the simple ladder appears in the Higgs propagator as well as in the gap equation. The momentum dependence of the four-Fermion vertex due to the higher dimensional operator in eq. (31) is not restricted to the s-channel. We get the same momentum dependence also in the t-channel. The gap equation therefore gets the same correction as the Higgs self-energy and we do not get a pole shift like in case of heavy scalar exchange. The higher dimensional operator becomes irrelevant in the low energy limit in agreement with the RG-argument.

\section{Conclusion}

Several questions in top-condensation which are quite difficult to handle by dynamical methods can be answered by extending the renormalization group (RG) approach to include higher dimensional operators. This method leads to constituent conditions different from the simplest case if additional relevant higher dimensional operators are introduced. It allows a clear and simple distinction between relevant and irrelevant operators. While relevant operators in the full theory change the constituent conditions and therefore the low energy predictions, the irrelevant operators are responsible for distinguishing the full theory from the effective theory near the cutoff scale. If irrelevant operators are absent, the model is just a reparametrization of its effective theory. This approach gives an intuitive formulation of the argument by Hasenfratz et al. and Zinn-Justin claiming that the standard model can always be reparametrized as a top-condensation model. It turns out that top-condensation by heavy scalar exchange is no theory in its own right but just a special case of a standard model reparametrization. The boundstate character of the Higgs has no physical significance since all operators of top-condensation are relevant at low energies. The specific role of the quadratic running in the RG-approach allows also the conclusion that the masses of vectorial boundstates in top-condensation cannot be lowered below the cutoff scale. Finally it 
can be seen that the replacement of a simple four-Fermion coupling by heavy gauge boson exchange does not introduce new relevant operators and therefore cannot change the pole conditions in the RG-approach. This guarantees that top-condensation by heavy vector boson interaction gives well controlled predictions for the top and the Higgs mass.

Acknowledgments: We would like to thank D. Kominis and M. Lindner for useful discussions and M. Lindner for helpful comments on the draft version of this paper.

This work was supported in part by the DFG under contract Li519/2-2.

\section{References}

[1] Y. Nambu and G. Jona-Lasinio, Phys. Rev. 122 (1961) 345;

V. G. Vaks and A. I. Larkin, Sov. Phys. JETP13 (1961) 192.

[2] Y. Nambu, New Theories in Physics, proceedings of the XI International Symposium on Elementary Particle Physics, edited by Z. Ajduk, S. Pokorski and A. Trautman (World Scientific, Singapore, 1989).

[3] V. A. Miranski, M. Tanabashi and K. Yamawaki, Mod. Phys. Lett. A4 (1989) 1043, Phys. Lett. B221 (1989) 177.

[4] W. A. Bardeen, C. T. Hill and M. Lindner, Phys. Rev. D41 (1990) 1647.

[5] A. Blumhofer, R. Dawid and M. Lindner, Phys. Lett. B360 (1995) 123.

[6] M. Lindner Int. J. Mod. Phys. A8 (1993) 2167;

G. Cvetic, DO-TH-97-03 (1997), hep-ph/9702381.

[7] A. Hasenfratz, P. Hasenfratz, K. Jansen, J. Kuti, Y. Shen, Nucl. Phys. B365 (1991) 79.

[8] J. Zinn-Justin, Saclay preprint SPhT/91-092.

[9] S. King and S. Mannan, Phys. Lett. B241 (1990) 249;

R. Bönisch, Phys. Lett. B268 (1991) 394;

M. Lindner and D. Ross, Nucl. Phys. B370 (1992) 30;

R. Dawid and S. Reznov, Phys. Lett. B388 (1996) 315.

[10] D. E. Clague and G. G. Ross, Nucl. Phys. B364 (1991) 43.

[11] M. Lindner and D. Lüst, Phys. Lett. B272 (1991) 91.

[12] U. Vogl and W. Weise, Prog. Part. Nucl. Phys. 27 (1991) 195.

[13] C. T. Hill, Phys. Lett. B266 (1991) 419.

[14] M. Luty, Phys. Rev. D41 (1990) 2893;

M. Suzuki, Phys. Rev. D41 (1990) 3457. 
[15] E. Akhmedov, M. Lindner, E. Schnapka and J. W. F. Valle, Phys. Rev. D53 (1996) 2752 .

[16] W. A. Bardeen, T. E. Clark and S. T. Love, Phys. Lett. B237 (1990) 235;

W. A. Bardeen, M. Carena, T. E. Clark, K. Sasaki and C. E. M. Wagner, Phys. Lett. B369 (1992) 33.

[17] M. Lindner, Z. Phys. C31 (1986) 295. 